\title{
Erratum to: Gender differences in colorectal cancer survival in Japan
}

\author{
Kenjiro Kotake $^{1} \cdot$ Michio Asano $^{2} \cdot$ Heita Ozawa $^{1} \cdot$ Hirotoshi Kobayashi $^{3}$. \\ Kenichi Sugihara ${ }^{3}$
}

Published online: 13 August 2015

(C) Japan Society of Clinical Oncology 2015

\section{Erratum to: Int J Clin Oncol \\ DOI 10.1007/s10147-015-0868-6}

Unfortunately, the original publication of the article contains an error in Fig. 1 and few errors in reference citations. The errors are corrected with this publication.

Few rows in Fig. 1 are not aligned properly. The corrected version of Fig. 1 is displayed here.

In the page 2, the citation of Ref. [6] in line 9 of left column should be [7], and the subsequent reference numbers should be increased by 1 .

In page 8 , the sentence "Similarly, some studies have reported differences in the administration of adjuvant chemotherapy between men and women [26-29]." should be written as "Similarly, some studies have reported differences in the administration of adjuvant chemotherapy between men and women [27-29]." That is the four references [26-29] should be changed to three references [27-29]. However, there is no change in the reference list.

The online version of the original article can be found under doi:10.1007/s10147-015-0868-6.

Kenjiro Kotake

kkotake@tcc.pref.tochigi.lg.jp

1 Department of Colorectal Surgery, Tochigi Cancer Center, 4-9-13 Yohnan, Utsunomiya, Tochigi 320-0834, Japan

2 Department of Surgery, Asano Clinic, 1628-1 Takatsuka-cho, Hamamatsu, Shizuoka 432-8061, Japan

3 Department of Surgical Oncology, Graduate School, Medical and Dental University, 1-5-45 Yushima, Bunkyo-ku, Tokyo 113-8510, Japan

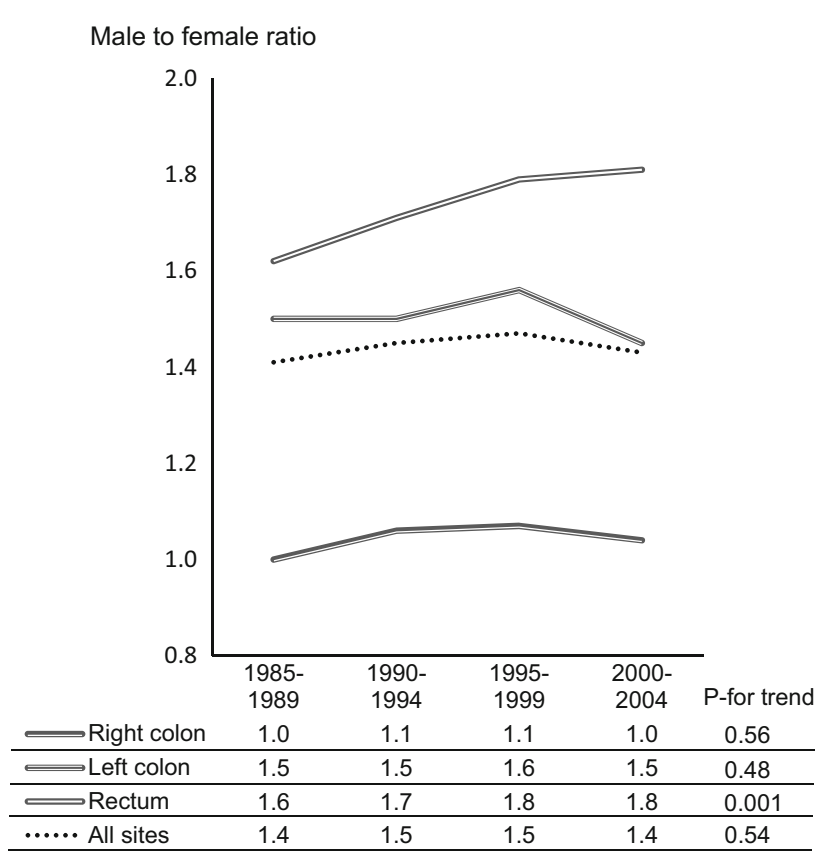

Fig. 1 Changes in the male to female ratio according to tumor subsite 